\section{Scaffold for the enlarged heart}

\section{By Lauren Martz, Staff Writer}

Cincinnati Children's Hospital Medical Center researchers have identified a scaffold protein, calcium and integrin binding 1, whose expression correlates with the development of cardiac hypertrophya condition for which only indirect treatments for related conditions are available. ${ }^{1}$ The protein's key selling point as a drug target is that it is upregulated only during pathologic hypertrophy; however, its lack of enzymatic activity could make it difficult to inhibit.

Cardiac hypertrophy-enlarged heartcan result from chronic hypertension, aortic stenosis or myocardial infarction (MI) and is a leading risk factor for heart failure. However, hypertrophy also is an important defense during exercise because it helps prevent damage due to increased stress and pressure. ${ }^{2}$

Rather than tease apart the mechanisms underlying the good and bad forms of hypertrophy, many companies have opted to treat the conditions that cause it, noted Anthony Muslin, director of the Center for Cardiovascular Research at the Washington University in St. Louis School of Medicine.

"There are treatment strategies for hypertension, which causes hypertrophy, such as diuretics, [angiotensin-converting enzyme] inhibitors and $\beta$-blockers, and there are therapeutic interventions for aortic stenosis, but there has not been a direct strategy to treat the enlargement of the heart muscle cells directly," said Muslin.

To identify new hypertrophy-associated genes and proteins, a group led by Joerg Heineke and Jeffery Molkentin performed a screen in cultured rat cardiomyocytes and identified calcium and integrin binding 1 (Cib1; calmyrin) as a gene that is upregulated following the induction of hypertrophy.

Molkentin is a professor of molecular cardiovascular biology at Cincinnati Children's Hospital and a Howard Hughes Medical Institute investigator. Heineke, a former member of Molkentin's team, now is a junior research group leader in the Department of Cardiology and Angiology at the Hannover Medical School.

In mice undergoing transverse aortic constriction (TAC), a procedure that induces both pathologic pressure overload of the heart and hypertrophy, Cib1 mRNA and protein levels were higher than those in controls. The protein also localized to the cardiomyocyte cell membranes in the pressure overloaded mice compared with control animals.

Cib1 knockout mice undergoing TAC had impaired cardiac growth, fewer induced hypertrophic marker genes and less fibrosis compared with wild-type mice, suggesting that blocking the target could help protect against hypertrophy.

The increase in CIB1 expression during hypertrophy was confirmed in human hypertrophic hearts compared with healthy hearts. Notably, the increase in expression did not occur in hearts undergoing physiological hypertrophy due to exercise, a finding that suggests increased CIB1 expression is specifically associated with pathogenic increases in myocardium size.

Finally, mechanistic studies revealed that CIB1's interaction with calcineurin is important in the development of cardiac hypertrophy.

Results were published in Nature Medicine. The team also included researchers from The University of North Carolina at Chapel Hill.

\section{Targeting CIB1}

"We now need to find ways to inhibit CIB1 either pharmacologically or, for example, through a gene therapy approach. Studies like this are underway in our lab," Heineke told SciBX.

Hitting the protein may prove problematic, said Muslin, because CIB1 is a scaffold protein without a catalytic site or enzymatic activity, making it difficult to develop a small molecule antagonist.

"CIB1 is not an enzyme. You therefore only have the option of knocking down its mRNA expression or inhibiting its binding. Historically, drugs that inhibit enzymes have been easier to develop. Fewer drugs are available that inhibit intracellular scaffold proteins or their binding interactions," he noted.

Junichi Sadoshima, vice chair of the Department of Cell Biology and Molecular Medicine at the University of Medicine and Dentistry of New Jersey, said it's not yet clear whether blocking that interaction would be sufficient to achieve a therapeutic effect.

"The authors did an excellent job, but they haven't yet shown that all of the CIB1 knockout phenotypic effects were a result of disrupting the interaction with calcineurin. It is possible that some of the beneficial effects of CIB1 knockout were due to calcineurinindependent mechanisms," said Sadoshima. "It will be important to prove that the effects occur exclusively through the interaction between calcineurin and CIB1 before developing and testing an inhibitor of the interaction."

Indeed, he said, "if calcineurin-independent mechanisms were found to contribute to the beneficial effects, it might be necessary to block expression of CIB1 in a heart-specific manner."

If this turns out to be the case, both Sadoshima and Muslin think the use of antisense or small interfering RNA would be the best platforms for therapeutic development. 


\section{ANALYSIS}

\section{TARGETS \& MECHANISMS}

\section{Toxicity concerns}

Another wrinkle is that CIB1 expression is ubiquitous throughout the body. Thus, even though CIB1 is upregulated during pathologic forms of hypertrophy, blocking the protein could have effects on cell growth or other critical processes in healthy tissues.

In addition, preclinical experiments have shown that although calcineurin has a role in hypertrophy, it is not an optimal target for that indication. For example, Muslin told SciBX that cyclosporine A, a generic calcineurin inhibitor, failed to reverse cardiac hypertrophy in human transplant recipients.

"Cyclosporine A also has renal toxicity," he noted. "It isn't completely clear whether this toxicity is an effect of inhibiting the calcineurin itself or because of an off-target activity of the drug such as causing reactive oxygen species generation. But past results in this pathway will warrant clear toxicity assessment of any new compound developed."

Muslin also said CIB1 interacts with other proteins such as sphingosine kinase and focal adhesion kinase. "This is a problem because it suggests that CIB1 probably does other things besides regulating the growth of heart muscle cells, so it is hard to know, given the complexity of its interacting partners, what could happen when its interactions are blocked or its expression is reduced," he said.

Sadoshima said the Nature Medicine paper provided some hints about the safety profile of Cib1 knockouts. Those animals, he said, had "defects in the reproductive system and angiogenesis. Obviously this is an issue suggesting that systemic delivery will cause side effects. They need to find a way to block this mechanism specifically in the context of cardiac hypertrophy."

Muslin thinks the best strategy might be to generate "small molecule inhibitors of the interaction between CIB1 and calcineurin at the cardiac muscle cell membrane." This could succeed where cyclosporine failed because inhibitors of the interaction would work via a different mechanism. The interaction inhibitors would prevent calcineurin from anchoring to membranes and signaling downstream genes that trigger hypertrophy. Cyclosporine only blocks the enzymatic activity of calcineurin.

To counteract any potential side effects of antagonizing CIB1 in general, both Sadoshima and Muslin think organ-specific delivery of CIB1 blockers will be important.

Heineke agreed. "Interfering with CIB1 for treatment of pathological cardiac hypertrophy will have to be targeted to the cardiomyocytes of the heart," he said. "This would be achievable with the use of specific gene therapy vectors that only reach cardiomyocytes."

He said the findings described in Nature Medicine are not available for licensing.

Martz, L. SciBX 3(30); doi:10.1038/scibx.2010.914

Published online Aug. 5, 2010

\section{REFERENCES}

1. Heineke, J. et al. Nat. Med.; published online July 18, 2010; doi:10.1038/nm.2181

Contact: Jeffery D. Molkentin, Cincinnati Children's Hospital Medical Center, Cincinnati, Ohio

e-mail: jeff.molkentin@cchmc.org

Contact: Joerg Heineke, Hannover Medical School, Hannover, Germany e-mail: Heineke.Joerg@mh-hannover.de

2. Levy, D. et al. JAMA 275, 1557-1562 (1996)

COMPANIES AND INSTITUTIONS MENTIONED

Cincinnati Children's Hospital Medical Center, Cincinnati, Ohio

Hannover Medical School, Hanover, Germany

Howard Hughes Medical Institute, Chevy Chase, Md.

University of Medicine and Dentistry of New Jersey, Newark, N.J.

The University of North Carolina at Chapel Hill, Chapel Hill, N.C.

Washington University in St. Louis School of Medicine,

St. Louis, Mo. 